\title{
Distortion Reduction in FIR Filters by Approximation through Window Factor on Function Coefficients
}

Sergio Bimbi Junior

Agenor de Toledo Fleury

Ronaldo Ruas

Vitor Chaves de Oliveira 


\title{
Distortion Reduction in FIR Filters by Approximation through Window Factor on Function Coefficients
}

\author{
Sergio Bimbi Junior, Agenor de Toledo Fleury, Ronaldo Ruas, Vitor Chaves de Oliveira \\ São Paulo State Technological Research Institute - IPT \\ Program - Master in Industrial Processes
}

\begin{abstract}
Filters are time-invariant linear systems which are able to modify the characteristics of the signals connected to their input, so that only a specific portion of the frequency components in a signal can reach the output of the filter. In dynamic systems, digital filters are applied in order to improve system measurements with regards to performance and stability. The present article demonstrates a modification in a low pass filters having Hamming window within the sample space $\pi$. In this development, the sample space $\pi$ is subdivided, wherein equation plots are added within a polynomial of order $n$. This technique provides the removal of unwanted frequency components in small angular frequency windows, providing the signal with acceleration towards the target when as compared to a low pass filter having Hamming window. In dynamic measurement systems, this feature is relevant, considering that the system shall have grater approximation to its target values, thus implementing an average which indicates the value being acquired in a more accurate and repetitive manner.
\end{abstract}

Key Words- Digital Filter, Digital Signal Processing, Low Pass Filter, High Pass Filter, Filter Pass Band, Band Reject Filter.

\section{INTRODUCTION}

Filters are time-invariant linear systems which are able to modify the characteristics of signals connected to their input, so that only a specific portion of the frequency components in a signal can reach the output of the filter. Considering analog signals $x(t)$ and $y(t)$ and a filter having impulse response function $h(t)$, as shown in Equation 1:

$$
y(t)=h(t) * x(t)(1)
$$

In the frequency domain, the equation can be solved as seen in Equation 2.

$$
Y(j \omega)=H(j \omega) * X(j \omega)(2)
$$

Assuming an implemented digital filter is a Digital Signal Processor (DSP) and the goal is to process an analog signal $x(t)$, a digital filter system can be characterized as shown in Figure 1 (21).

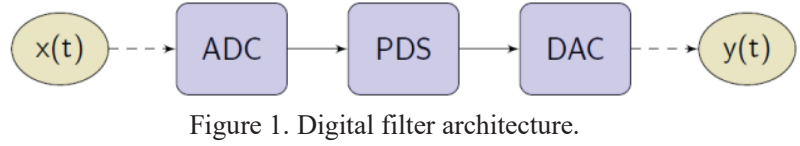

If the signals to be processed are digital, the diagram can be shown in summary form, as seen in Figure 2 (21).

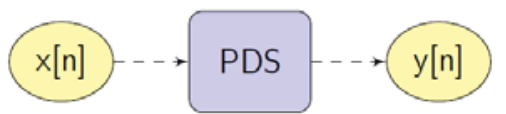

Figure 2. Digital filter architecture in summary form.

In order to implement a digital and time-invariant (LTI) filter, a DSP is required, in which computational algorithms are arranged. These can be represented in the form of block diagrams using basic structures such as unit delays, gains, adders, feedbacks and delay structures in the block diagram, which is similar to the order of difference equations (transfer function) of the filter known as Canonical structure.

\section{DIGITAL FILTERS VERSUS ANALOG FILTERS}

The comparison relationship between an analog and a digital filter is directly linked to the complexity, precision and design adaptability (22). An analog filter is economically more recommended than a digital filter, but once the system is implemented, due to its discrete components, the system becomes too complex for adaptations and improvements. Another interesting feature of analog filters, given that the system is implemented by external components, is the change in the characteristics of these devices with respect to environmental factors such as temperature and humidity, thus compromising their best theoretical performance. In turn, digital filters, with respect to Analog to Digital conversion (AD) and Digital to Analog (DA) conversion and the processing itself, have a poorer response time as compared to the analog filter. Digital filters also have errors inherent to the quantization process (performed in $\mathrm{AD}$ conversion) and rounding errors due to the use of digital words having finite length. In recursive filters, this phenomenon can lead to greater instability (23). On the other hand, the ability to implement this filter within a processed system leads to greater flexibility in the updating and maintenance of the 
project. The architecture of a digital filter can be seen in Figure 1.

\section{IIR FILTER}

Infinite Impulse Response (IIR) filters are those which have an infinitely long-lasting response to an impulse and having recursive nature, thus, it can be concluded that this filter is characterized by relying on both the current input and the previous input. Figure 3 illustrates the structure for developing an IIR filter, function $x(k)$ is the input signal, values $a 1$ to $a Q$ and $b 0$ to $b P$ are coefficients representing the type of filtering that is being performed (high-pass, low-pass, band-pass) and function $y(k)$ is the output signal as a result of the filtering of signal $x(k)(24)$.

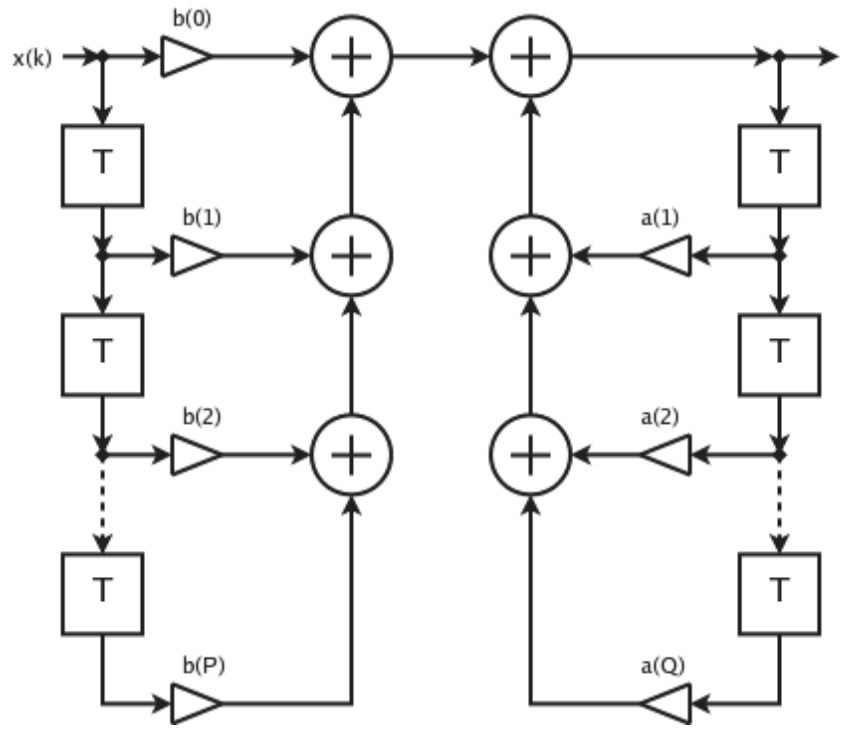

Figure 3. Structure for developing a digital IIR filter.

In mathematical aspects, the output of a digital IIR filter is represented recursively as seen in Equation 3, where $a k$ and $b k$ are filter coefficients.

$$
\begin{gathered}
y[n]=\sum_{k=0}^{\infty} \mathrm{h}[\mathrm{k}] *[\mathrm{n}-\mathrm{k}]= \\
\sum_{k=0}^{n} b_{k} *[n-k]= \\
\sum_{k=1}^{M} a_{k} y *[n-k]=(3)
\end{gathered}
$$

By applying the z-transform we obtain the transfer functions shown in Equation 4.

$$
H(z)=\frac{Y(z)}{X(z)}(4)
$$

By breaking down $Y(z)$ and $X(z)$, the transfer function equation in an IIR filter is obtained as seen in Equation 5.

$$
H(z)=\frac{\sum_{k=0}^{N} b_{k} z^{-k}}{1+\sum_{k=1}^{M} a_{k} z^{-k}}(5)
$$

\section{FIR FILTER}

Finite Impulse Response (FIR) filters are those which show a finitely long-lasting impulse response (25). This filter is characterized by a discrete transfer function which can be seen in Equation 6.

$$
\frac{Y(z)}{X(z)}=\frac{\sum_{k=0}^{M} a_{k} z^{(M-k)}}{Z^{M}}(6)
$$

This discrete function can be rewritten as a polynomial function of negative powers of $z$. FIR filters have welldefined characteristics, which are:

1) Finite memory, which stipulates that any transient has limited duration.

2) Always developed as stable Bounded Input, Bounded Output (BIBO).

3) It is possible to develop a desired magnitude response having linear phase response.

In Figure 4, the structure of an FIR filter can be seen.

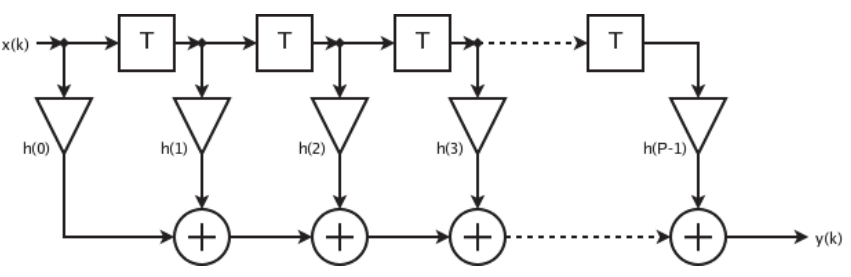

Figure 4.Structure for developing a digital FIR filter.

\section{FILTER OR FIR IIR}

The decision to evaluate which filter is the best for the application, i.e., whether to use an FIR filter or an IIR filter, is directly connected to project-specific features. FIR filters have linear phase response, which implies that no phase distortion is produced in the filtered signal. This feature is important in applications such as audio and image processing, biomedicine and data transmission (26).

FIR filters are developed in non-recursive mode, thus they are always stable. This characteristic cannot be guaranteed for IIR filters. The effects of finite precision and quantization errors are less severe for FIR filters (27).

IIR filters generally require less coefficients than FIR in order to meet the same design specification. A lower order filter has a shorter runtime. Analog filters may be converted to IIR digital filters fairly easily. In general, one can use an IIR filter when the largest relevance is a very selective response in the frequency domain, or when the implementation an analog filter is required. FIR filters may be used when the number of coefficients is not very high (FIR structure stability is guaranteed), and especially when the desired phase distortion is small (28).

\section{DESIGNING DIGITAL FILTERS}

Digital Filter designs consist in determining a transfer function that fits the frequency response specifications 
required for a particular project or application. Within filter applications, elements with well-defined characteristics exist:

1) Low-Pass Filter (LPF).

2) High-Pass Filter (HPF).

3) Band-Pass Filter (BPF).

4) Band-Stop Filter (BSF).

Impulse responses to the ideal transfer functions are not digitally achievable given that they have infinite length and are not causal. For FIR filters, one approach used is the truncation of the impulse response of ideal filters (29).

For IIR filters, it is possible to map transfer functions originally designed to analog filters to the $\mathrm{z}$ domain.

\section{APPROXIMATION FOR FIR FILTERS}

For implementing a filter, it is necessary to develop from its transfer function, as shown in Equation 7.

$$
H[z]=\sum_{n=-\infty}^{\infty} \mathrm{h}[\mathrm{n}] z^{-n}(7)
$$

However, its behavior is best characterized as a function of its frequency response as shown in Equation 8.

$$
H\left[e^{j \omega}\right]=\sum_{n=-\infty}^{\infty} \mathrm{h}[\mathrm{n}] e^{-j \omega n}(8)
$$

Whereas $h[n]$ can be shown by Equation 9 .

$$
h[n]=\int_{-\pi}^{\pi} \mathrm{H}\left(e^{j \omega}\right) e^{j \omega} d \omega(9)
$$

With these determinations, $H\left(e^{j \omega}\right)$ and $h[n]$ pairs are obtained for ideal filters shown in Table 1. 


\begin{tabular}{|c|c|c|}
\hline Filter type & $\begin{array}{c}\text { Response } \\
\text { magnitude } \\
\left|H\left(e^{j \omega}\right)\right|\end{array}$ & $\begin{array}{c}\text { Impulse } \\
\text { response } h[n]\end{array}$ \\
\hline LPF & $\begin{array}{l}1 \text { for } 0 \leq|\omega| \leq \omega_{c} \\
0 \text { for } \omega_{c}<|\omega| \leq \pi\end{array}$ & $\begin{array}{c}\frac{\omega_{c}}{\pi} \text { for } \mathrm{n}=0 \\
\frac{1}{\pi n} \operatorname{Sin}\left(\omega_{c} n\right) \text { for } \mathrm{n} 0 \neq\end{array}$ \\
\hline HPF & $\begin{array}{l}0 \text { for } 0 \leq|\omega| \leq \omega_{c} \\
1 \text { for } \omega_{c}<|\omega| \leq \pi\end{array}$ & $\begin{array}{c}\frac{\omega_{c}}{\pi} \text { for } \mathrm{n}=0 \\
-\frac{1}{\pi n} \sin \left(\omega_{c} n\right) \text { for } \mathrm{n} \neq \\
0\end{array}$ \\
\hline BPF & $\begin{array}{c}0 \text { for } 0 \leq|\omega| \leq \omega_{c 1} \\
1 \text { for } \omega_{c 1} \leq|\omega| \leq \\
\omega_{c 2} \\
0 \text { for } \omega_{c 2}<|\omega| \leq \pi\end{array}$ & $\begin{array}{c}\frac{\omega_{c 2}-\omega_{c 1}}{\pi} \text { for } \mathrm{n}=0 \\
-\frac{1}{\pi n}\left[\operatorname{SIN}\left(\omega_{c 2} n\right)-\right. \\
{\left[\operatorname{SIN}\left(\omega_{c 1} n\right)\right] \text { for } \mathrm{n} \neq} \\
0\end{array}$ \\
\hline BSF & $\begin{array}{c}1 \text { for } 0 \leq|\omega| \leq \omega_{c 1} \\
0 \text { for } \omega_{c 1} \leq|\omega| \leq \\
\omega_{c 2} \\
1 \text { for } \omega_{c 2}<|\omega| \leq \pi\end{array}$ & $\begin{array}{c}1-\left(\frac{\omega_{c 2}-\omega_{c 1}}{\pi}\right) \text { for } \mathrm{n}= \\
0 \\
\frac{1}{\pi n}\left[\sin \left(\omega_{c 1} n\right)-\right. \\
{\left[\sin \left(\omega_{c 2} n\right)\right] \text { for } \mathrm{n} \neq} \\
0\end{array}$ \\
\hline
\end{tabular}

Table 1. Characteristics of ideal filters.

With Table 1 specifying the response behavior in magnitude and the impulse response, one is able to check the behavior of LPF, HPF, BPF and BSF filters respectively in Figures 5, 6, 7 and 8 .

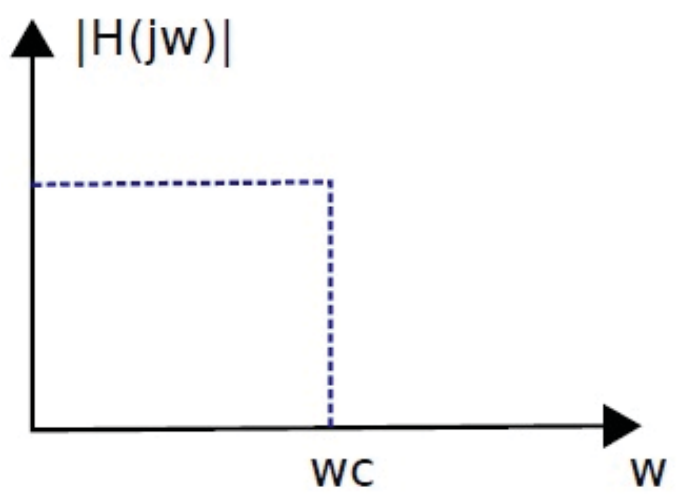

Figure 5. LPF behavior in the frequency domain.

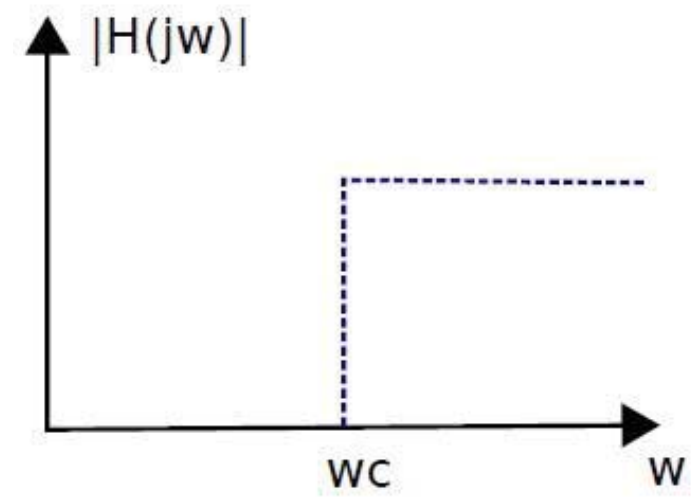

Figure 6. HPF behavior in the frequency domain.

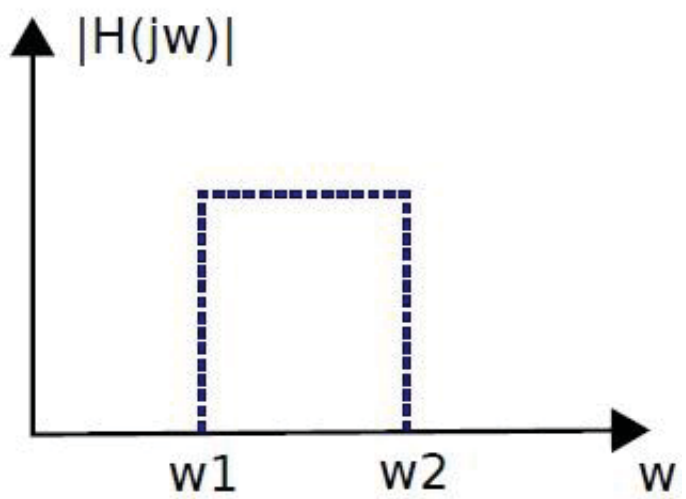

Figure 7. BPF behavior in the frequency domain.

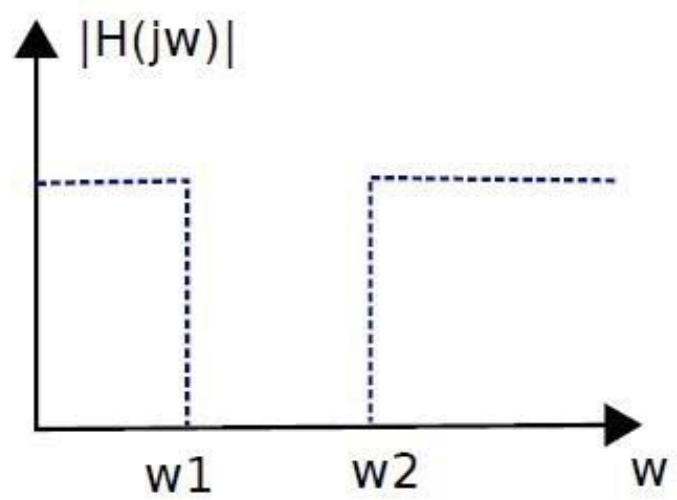

Figure 8 . BSF behavior in the frequency domain.

\section{APPROXIMATION FOR FIR FILTERS BY USING WINDOW FUNCTIONS}

One way to have a better performance and overcome limitations inherent to the realization of the impulse response function $h[n]$ of ideal filters is to define an auxiliary sequence $h^{\prime}(n)$ having a finite length of order $M$ as described in Equation 10.

$$
h^{\prime}(n)=\left\{\begin{array}{c}
\left\{H[n] W[n], \quad|n| \leq \frac{M}{2}\right. \\
0,|n|>\frac{M}{2}
\end{array}\right.
$$

The obtained response, despite having finite length, is not causal. However, it can be transformed into causal by multiplying by $z^{-M / 2}$, without distorting the magnitude response and without destroying the linear phase property. Sequence $w[n]$ is known as the "window function" (30). 


\section{FIR FILTERS USING WINDOW}

The central idea of digital filter design by using windows is to select a suitable filter having an ideal frequency (which is always non-causal and has infinite impulse response) and then truncate its impulse response in a window in order to get a causal FIR, linear phase filter. Considering an ideal low pass filter $H d\left(e^{j w}\right)$ having magnitude 1 and linear phase with a pass band and zero response at the cutoff band, as seen in Equation 11 (31):

$$
H d\left(e^{j w}\right)=\left\{\begin{array}{c}
1 \cdot e^{-j \alpha w}, \quad|W| \leq W_{C} \\
0, W_{C}<|W| \leq \pi
\end{array}\right.
$$

The impulse response of this filter is infinite, given by Equation 12 and 13.

$$
\begin{gathered}
h d[n]=\mathcal{J}^{-1}\left[H_{d}\left(e^{j w}\right)\right]=\frac{1}{2 \pi} \int_{-\pi}^{\pi} H_{d}\left(e^{j \omega}\right) e^{j \omega} d \omega(12) \\
h d[n]=\frac{1}{2 \pi} \int_{-\omega_{c}}^{\omega_{c}} 1 \cdot e^{j \omega} e^{j \omega} d \omega=\frac{\operatorname{sen}\left[\omega_{c}(n-\alpha)\right]}{\pi(n-\alpha)}
\end{gathered}
$$

In order to obtain a causal linear phase FIR filter $h[n]$ of length $M$, as shown in Equation 14.

$$
h(n)=\left\{\begin{array}{ccc}
h_{d}[n], & 0 \leq n \leq M-1 \\
0, & \text { else }
\end{array}\right.
$$

Where $\alpha$ is represented by Equation 15 .

$$
\alpha=\frac{(M-1)}{2}(15)
$$

This operation is called windowing. In general, $h[n]$ may be characterized by the product of $H_{d}[n]$ in a window $w[n]$ as shown in Equation 16.

$$
h[n]=H_{d}[n] . w[n](16)
$$

Where $w[n]$ is a symmetric function with respect to $\alpha$ within the range $0 \leq n \leq M-1$, with zero being absent from this range. It is useful to mention that, depending on how $w[n]$ is obtained, different filter techniques can be obtained as shown in Equation 17, defining a rectangular window (32).

$$
w(n)=\left\{\begin{array}{c}
1, \quad 0 \leq n \leq M-1 \\
0, \quad \text { else }
\end{array}\right.
$$

Thus, it is necessary to define a finite $h[n]$ sequence for the digital filter from $H_{d}[n]$. The Fourier transform is a discrete sequence in time, whereas $H\left(e^{j w}\right)$ is continuous. The causal FIR filter $H\left(e^{j w}\right)$ response is obtained in the frequency domain by the convolution of $H\left(e^{j w}\right)$ and the window response $W\left(e^{j w}\right)$, as shown in Equation 18.

$$
\begin{aligned}
& H\left[e^{j \omega}\right]=H\left(e^{j w}\right) * W\left(e^{j w}\right) \\
& =\frac{1}{2 \pi} \int_{-\pi}^{\pi} \mathrm{W}\left(e^{j \omega}\right) H_{d}\left(e^{j(w-\lambda}\right) d \lambda(18)
\end{aligned}
$$

\section{DISTORTION REDUCTION WITHIN WINDOW IN AN FIR FILTER WITH POLYNOMIAL APPLIED IN RADIANS}

The distortion reduction within the window performed in a filter is an important factor with regards to rejection of unwanted points in present lobes, which are not acceptable beyond the main lobe; this is because they represent frequencies that are not required for that filter. As the number of $M$ coefficients increases, the width of each sidelobe decreases, but the area over them remains constant. This causes ripples to experience peaks near band edges. This fact is referred to as "Gibbs Phenomenon."

In order to perform the proposed distortion reduction within one window out of the many applicable, adaptation of the window equation is necessary; in this article, the window used is the Hamming window shown in Equation 19, where $n$ is equal to the current filter coefficient and $M$ is the total number of coefficients.

$$
w[n]=0,54-046 \cos \left(\frac{2 \pi n}{M}\right)
$$

The $2 \pi$ factor represents the entire spectrum of radian axes. The technique enables the inclusion of new points to the windowing; this occurs by dividing the $2 \pi$ radian spectrum into smaller points in order to obtain a more efficient filter. This takes place with the implementation of an $n$-th polynomial applied to $2 \pi$, in addition to the 0.46 factor in order to maintain linearity. In Figure 9 and 10, respectively, the full radian axis and the division of the total radian spectrum can be viewed (33).

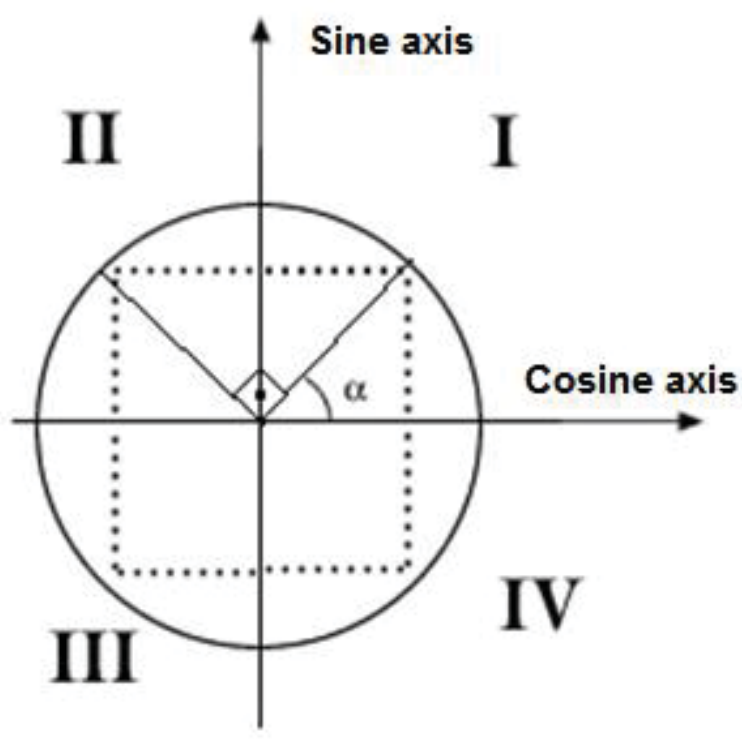

Figure 9. Full $2 \pi$ radian spectrum. 


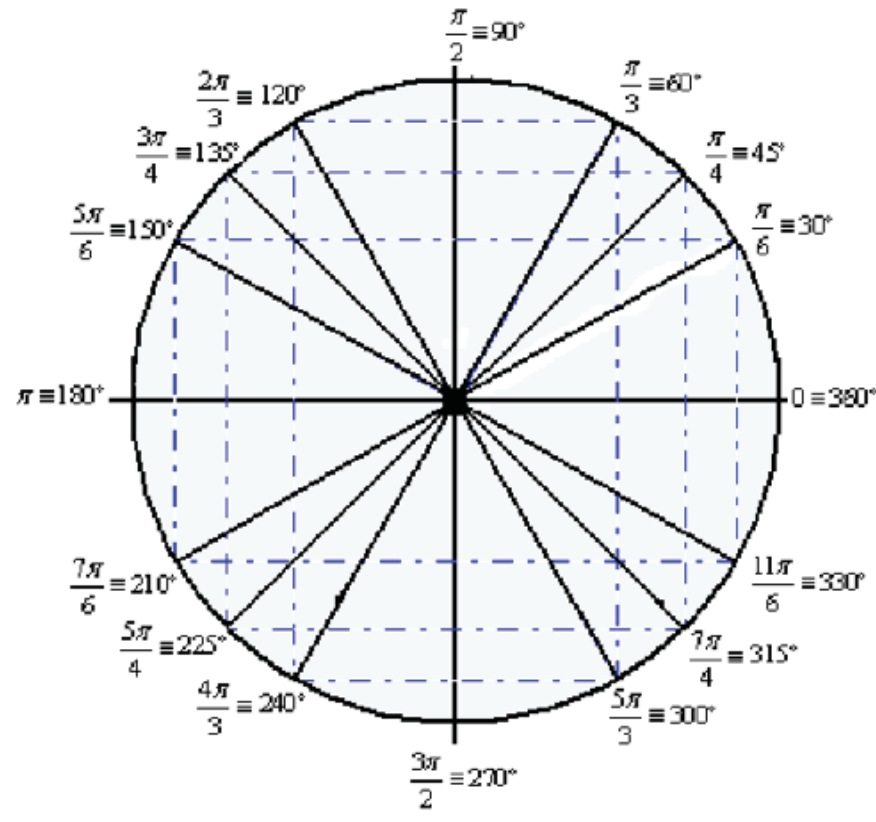

Figure 10. Full $2 \pi$ radian spectrum divided into smaller points.

The central focus of this process is to minimize the effect of lower lobes not included in the cutoff frequency determined within the system, which consists of the sum of window $w[n]$ in a polynomial having factor $n x-1$ for $n x \leq 1$. The described implementation can be seen in Equation 20.

$$
\begin{gathered}
w[n]=\left[0,54-0,46^{n x-1} \cos \left(\frac{2^{n x-1} \pi n}{M}\right)\right] \\
+\left[0,54-0,46^{n x-2} \cos \left(\frac{2^{n x-2} \pi n}{M}\right)\right] \\
+\left[0,54-0,46^{n x-3} \cos \left(\frac{2^{n x-3} \pi n}{M}\right)\right] \\
+\left[0,54-0,46^{n x-m x} \cos \left(\frac{2^{n x-m x} \pi n}{M}\right)\right](20)
\end{gathered}
$$

Equation 20 describes the sum of a polynomial applied to $w[n]$ applied to a Hamming window as an example.

In Figures 11 and 12, respectively, the driving windows can be seen in the time domain, applied to Equation 19 and 20 , respectively.

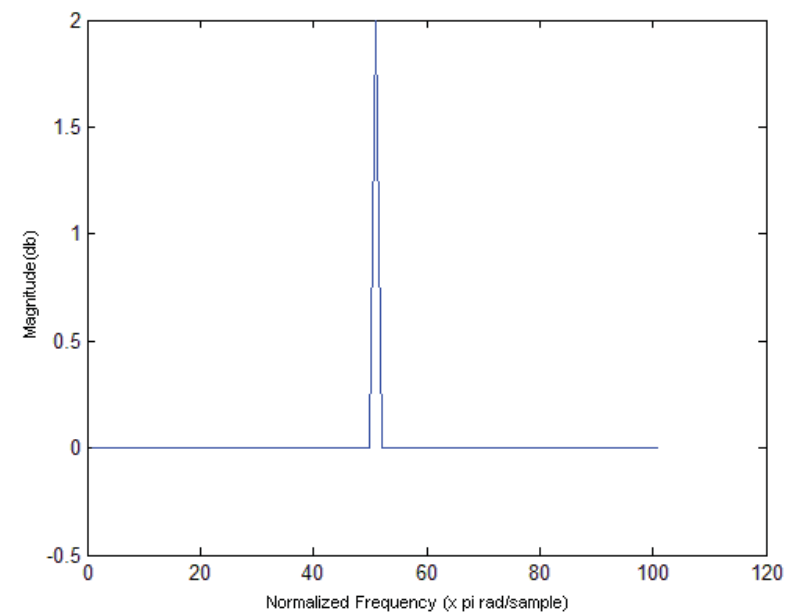

Figure 11. Conduction window in the time domain without changing w[n] (Equation 19).

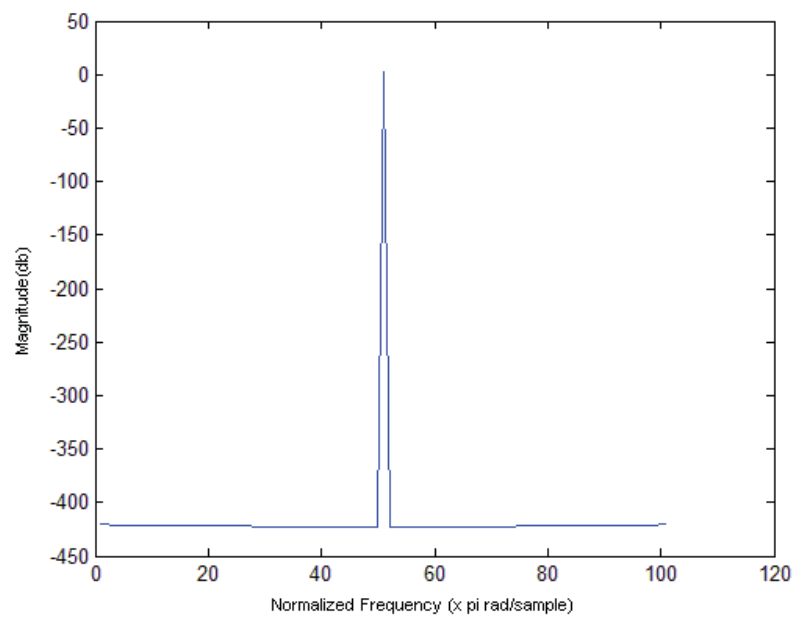

Figure 12. Conduction window in the time domain when changing $w[n]$ (Equation 20).

By applying the discrete Fourier transform, one is able to verify the behavior of both implementations in the frequency domain. In Figure 13, the filter behavior in the frequency domain can be seen; it is possible to check the pass region and cut-off frequency as a function of system specifications. One can also verify peaks at band regions, or "Gibbs phenomenon" (34).

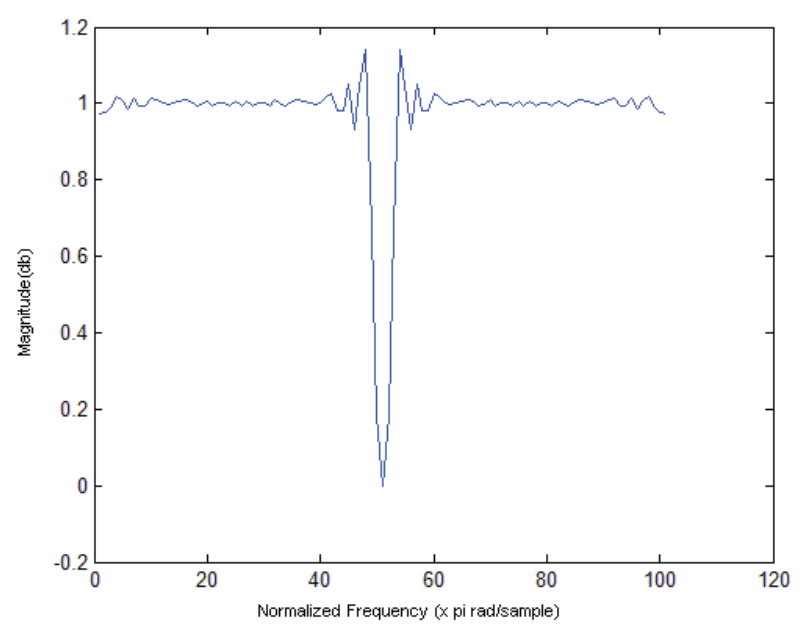

Figure 13. Filter having Hamming window in the frequency domain, with $w[n]$ unchanged.

In Figure 14, the filter behavior in the frequency domain can be seen, with a pass frequency region better defined at its cutoff frequency, as well as the reduction in "Gibbs phenomenon" effects, where it is present in the lower than zero region, so that, by applying the modulus, this phenomenon will be eliminated. 


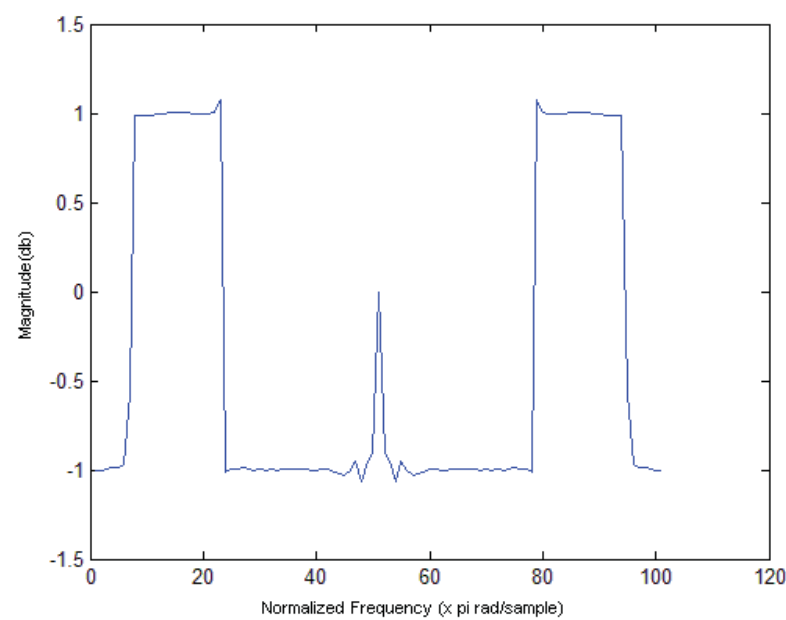

Figure 14. Filter having Hamming window in the frequency domain with changes in $w[n]$.

\section{EQUATION APPLICATION IN THE TIME DOMAIN}

The development of a low pass filter with Hamming window computationally consists of the implementation of a "First In - First Out", wherein its coefficients are accumulated in the time domain and multiplied by the calculated filter coefficient. The example shown in Figure 15 consists of a low pass filter having Hamming window, with a cutoff frequency of $1 \mathrm{~Hz}$ and $100 \mathrm{~Hz}$ sampling rate. To that filter, 136 coefficients are applied to a signal that emulates a harmonic developed by using an approximation sequence of the number $\pi$.

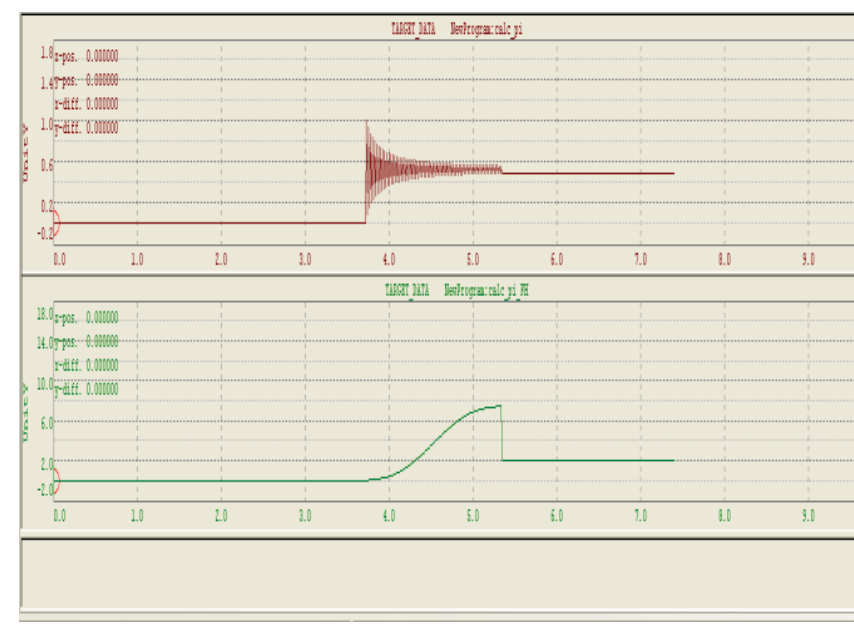

Figure 15. Filter having Hamming window in the time domain.

Figure 16 shows the same computational implementation mentioned in Figure 14 with the modification of the coefficients calculated in accordance with Equation 20 (modified Hamming - Bimbi). This will demonstrate the increased linearity and speed of convergence to the target point.

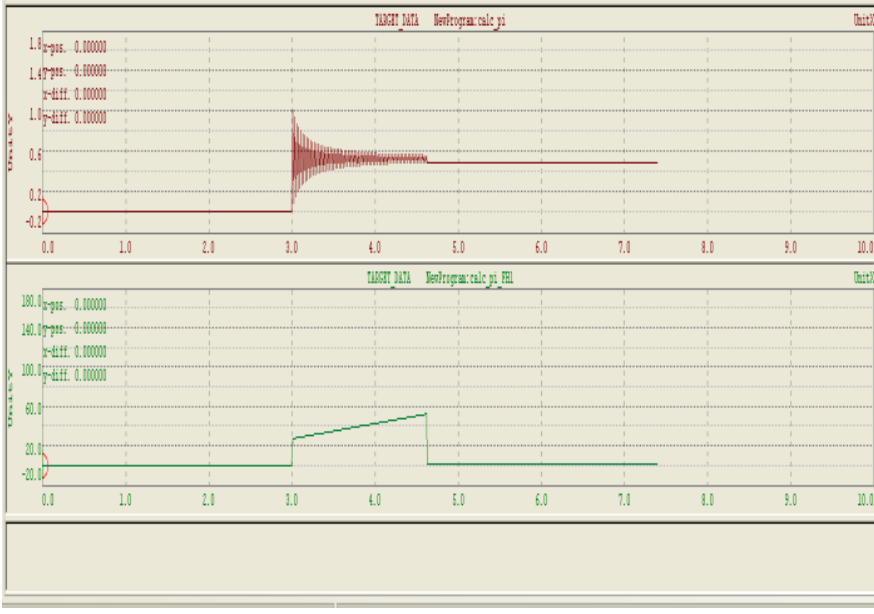

Figure 16. Filter having modified Hamming window in the time domain.

\section{CONCLUSION}

The inclusion of a modification in Equation 19, turning it into Equation 20, provides benefits in the implementation of digital filters having windowing, which one is able to verify in the plots shown in Figures 13 and 14 with respect to the frequency spectrum, in addition to a reduction of the "Gibbs phenomenon". The minimization of spurious frequencies with the polynomial calculation within the filter window minimizes the passing of spurious frequencies and enables the reduction of the number of coefficients applied; this aspect computationally reduces processing time in what we envision as dynamic systems that require limited time, such as dynamic measurement systems. From a time domain point of view, one can verify the increase in response time and linearity with regards to the faster convergence of the obtained reading towards the actual value to be measured, thus removing spurious frequencies from these samples. 


\section{REFERENCES}

[1] Adams, J.W. (1991a). FIR digital filters with least-squares stopbands subject to peak-gain constraints. IEEE Transactions on Circuits and Systems, 39, 376-88.

[2] Adams, J. W. (1991b). A new optimal window. IEEE Transactions on Signal Processing,39, 1753-69.

[3] Adams, J.W. \& Willson, Jr., A. N. (1983).Anew approach to FIR digital filters with fewer multipliers and reduced sensitivity. IEEE Transactions on Circuits and Systems, CAS-30, 277-83.

[4] Adams, J.W. \&Willson, Jr., A. N. (1984). Some efficient digital prefilter structures. IEEE Transactions on Circuits and Systems, CAS-31, 260-5.

[5] Ahmed, N., Natarajan,T.\&Rao, K. R. (1974). Discrete cosine transform. IEEE Transactionson Computers, C-23, 90-3.

[6] Akansu, A. N. \& Medley, M. J. (1999). Wavelets, Subband and Block Transforms in Communications and Multimedia. Boston, MA: Kluwer Academic Publishers.

[7] Antoniou,A. (1982). Accelerated procedure for the design of equiripple nonrecursive digital filters. IEE Proceedings - Part $G, 129,1-10$.

[8] Antoniou, A. (1983). New improved method for design of weighted-Chebyschev, nonrecursive, digital filters. IEEE Transactions on Circuits and Systems, CAS-30, 740-50.

[9] Antoniou, A. (1993). Digital Filters: Analysis, Design, and Applications, 2nd edn. New York, NY: McGraw-Hill.

[10] Antoniou, A. (2006). Digital Signal Processing: Signals, Systems, and Filters. New York, NY: McGraw-Hill.Antoniou, A. \& Lu, W.-S. (2007). Practical Optimization: Algorithms and Engineering Applications. New York, NY: Springer.

[11] Antoniou, A. \& Rezk, M. G. (1977). Digital filters synthesis using concept of generalizedimmitance converter. IEE Journal of Electronics Circuits, 1, 207-16. Antoniou, A. \& Rezk, M. G. (1980).Acomparison of cascade and wave fixed-point digitalfilter structures. IEEE Transactions on Circuits and Systems, CAS-27, 1184-94.

[12] Apostol, T. M. (1967). Calculus, 2nd edn., volume I. Toronto: Xerox College Publishing. Avenhaus, E. (1972). On the design of digital filters with coefficients of limited wordlength. IEEE Transactions on Audio and Electroacoustics, AU-20, 206-12. Bauer, P. H. \& Wang, J. (1993). Limit cycle bounds for floatingpoint implementations of second-order recursive digital filters. IEEE Transactions on Circuits and Systems II: Analog and Digital Signal Processing, 39, 493-501. Corrections on 41, 176 February 1994.

[13] Belevitch, V. (1968). Classical Network Theory. San Francisco, CA: Holden-Day.

[14] Benvenuto, N., Franks, L. E. \& Hill, Jr., F. S. (1984). On the design of FIR filters with power-of-two coefficients. IEEE Transactions on Communications, COM-32, 1299-307.

[15] Bhaskaran, V. \& Konstantinides, K. (1997). Image and Video Compression Standards: Algorithms and Architectures. Boston, MA: Kluwer Academic Publishers. Bomar, B.W. (1989). On the design of second-order state-space digital filter sections. IEEE Transactions on Circuits and Systems, 36, 542-52.

[16] Bomar, B.W. \& Joseph, R. D. (1987). Calculation of $L \infty$ norms in second-order state-space digital filter sections. IEEE Transactions on Circuits and Systems, CAS-34, 983-4.

[17] Bomar, B.W., Smith, L. M. \& Joseph, R. D. (1997). Roundoff noise analysis of state-space digital filters implemented on floating-point digital signal processors. IEEE Transactions on Circuits and Systems II: Analog and Digital Signal Processing, 44, 952-5.

[18] Boyd, S. \& Vandenberghe, L. (2004). Convex Optimization. Cambridge, UK: Cambridge University Press. Bracewell, R. N. (1984). The fast Hartley transform. Proceedings of the IEEE, 72, 1010-18.

[19] Bracewell, R. N. (1994). Aspects of the Hartley transform. Proceedings of the IEEE, 82, 381-6.

[20] Burrus, C. S. \& Parks, T. W. (1970). Time domain design of recursive digital filters. IEEE Transactions on Audio and Electroacoustics, AU-18, 137-41.

[21] Butterweck, H. J. (1975). Suppression of parasitic oscillations in second-order digital filters by means of a controlled-rounding arithmetic. Archiv Elektrotechnik und Übertragungstechnik, 29, $371-4$

[22] Butterweck, H. J., van Meer, A. C. P. \& Verkroost, G. (1984). New second-order digital filter sections without limit cycles. IEEE Transactions on Circuits and Systems, CAS-31, 141-6.
[23] Cabezas, J. C. E. \& Diniz, P. S. R. (1990). FIR filters using interpolated prefilters and equalizers. IEEE Transactions on Circuits and Systems, 37, 17-23.

[24] Chang, T.-L. (1981). Suppression of limit cycles in digital filters designed with one magnitude-truncation quantizer. IEEE Transactions on Circuits and Systems, CAS-28, 107-11.

[25] Charalambous, C. \& Antoniou, A. (1980). Equalisation of recursive digital filters. IEE Proceedings - Part G, 127, 219-25. 865 References

[26] Chen, W.-H., Smith, C. H. \& Fralick, S. C. (1977). A fast computational algorithm for the discrete cosine transform. IEEE Transactions on Communications, COM-25, 1004-9.

[27] Cheney, E.W. (1966). Introduction to Approximation Theory. NewYork,NY: McGraw-Hill.

[28] Churchill, R.V. (1975). Complex Variables and Applications NewYork,NY: McGraw-Hill.

[29] Claasen, T. A. C. M., Mecklenbrauker,W. F. G. \& Peek, J. B. H. (1975). On the stability of the forced response of digital filters with overflow nonlinearities. IEEE Transactions on Circuits and Systems, CAS-22, 692-6.

[30] Cochran, W., Cooley, J., Favin, D. et al. (1967). What is the fast Fourier transform? IEEE Transactions on Audio and Electroacoustics, AU-15, 45-55.

[31] Cohen, A., Daubechies, I. \& Feauveau, J. C. (1992). Biorthogonal bases of compactly supported wavelets. Communications on Pure and Applied Mathematics, XLV, 485560

[32] Constantinides, A. G. (1970). Spectral transformations for digital filters. IEE Proceedings, 117, 1585-90.

[33] Cooley, J.W. \&Tukey, J.W. (1965).An algorithm for the machine computation of complex Fourier series. Mathematics of Computation, 19, 297-301.

[34] Cortelazzo, G.\&Lightner, M. R. (1984). Simultaneous design of both magnitude and group delay of IIR and FIR filters based on multiple criterion optimization. IEEE Transactions on Acoustics, Speech, and Signal Processing, ASSP-32, 9949-67.

Sergio Junior Bimbi (e-mail: sergio.bimbi@fatec.sp.gov.br) is pursuing a Master's degree in Electrical Engineering (IPT) and holds a Bachelor's degree in Telecommunications Engineering from the FIEO University Center. He is currently an Electronics Engineer at Masipack and University Professor at the São Paulo State Technological College (FATEC). Sergio has experience in Electrical Engineering with emphasis in Embedded Electronics and Telecommunications, developing hardware for numerical controls, industrial automation and VHDL programming. He has developed research at the UNIFIEO University Center with FPGAs that are currently used in many subsequent projects. He has expertise with developing measurement systems, automatic weighing scales, PLC programming, control systems, weight checkers, digital filters for the field of measurements, etc. He develops research in the field of SDR (QPSK) with ZYNQ family integrated circuits having dynamic partial reconfiguration.

Vitor Chaves de Oliveira (e-mail: vitor.c.br@ieee.org) is a doctoral candidate in Electrical Engineering (Mackenzie), Master in Electrical Engineering (PUCC), attending specialization in Electrical Engineering and Power Systems (UNISAL), Bachelor of Computer Science (UNISAL) and Computer Technician (SENAI). University Professor working in the fields of Electrical Engineering and Computer Science; Magazine editor for the Brazilian Society of Television Engineering (SET); IT/ERP/Telecom Infrastructure Consultant at Coach IT Consulting. He is an author of books in the cloud computing field and has published dozens of articles in scientific journals and at national and international conferences. His research interests are focused on Telecommunications: IP networks, Radio 
Broadcasting (Digital TV), Electromagnetic Theory, Control Algorithms and Mathematical Modeling of Systems.

Agenor de Toledo Fleury (e-mail: agenor.fleury@poli.usp ) Bachelor of Mechanical Engineering from ITA / Aeronautics Technological Institute (1973), Master of Mechanical Engineering from the University of São Paulo (1978) and $\mathrm{PhD}$ in Mechanical Engineering from the University of São Paulo (1985). He is currently a professor at FEI University Center, where he is Coordinator for the Mechanical Engineering Graduate Program and a $\mathrm{PhD}$ professor at the Polytechnic School of the University of São Paulo. He has experience in various projects related to Mechanical Engineering, with emphasis on Dynamic and Control Systems. His most recent projects address the modeling and control of nonlinear systems, optimal control and estimation of dynamic system states in Biomechanics, Robotics, Automotive Engineering and Embedded Systems applications. He received the SAE Brazil 2010 Engineering Education Award.

Ronaldo Ruas (e-mail: ronaldo.ruas@fatec.sp.gov.br) Bachelor's degree in Material Processes and Electronic Components from São Paulo State Julio de Mesquita Filho University (1998). Master's degree in Electrical Engineering from the University of São Paulo (2001). PhD in Electrical Engineering from the University of São Paulo (2006). Currently collaborates as a guest researcher at the integrated systems laboratory (LSI), in the plasmas and new materials field, at the Polytechnic School of the University of São Paulo. He works at Protec Brazil as a technical manager in the development of plasma-assisted thin film processes (PVD, PECVD). He teaches courses in the fields of electronics, Microprocessors and Applied Electricity at the Osasco Technological College, where he is a coordinator for the Bachelor of Industrial Automation Technology course. 